\title{
Isolation and Protein Profiling of Outer Membrane Proteins (OMPS) of Salmonella typhi
}

\author{
Yashpal Singh $^{1}$, Anjani Saxena ${ }^{2}$, Rajesh Kumar $^{3}$ and M.K. Saxena ${ }^{4^{*}}$
}

${ }^{I}$ Department of Molecular Biology and Genetic Engg, College of Basic Science \& Humanities, G.B. Pant University of Agriculture \&Technology, Pantnagar, Uttarakhand, India

${ }^{2}$ Department of Pharmacology \& Toxicology, ${ }^{3}$ Department of Veterinary Microbiology, ${ }^{4}$ Department of Veterinary Physiology and Biochemistry, College of Veterinary and Animal Sciences, G.B. Pant University of Agriculture \&Technology, Pantnagar, Uttarakhand, India

*Corresponding author

\section{A B S T R A C T}

\section{Keywords}

Salmonella, Outer membrane proteins, vaccine

Article Info

Accepted:

15 July 2018

Available Online:

10 August 2018
Salmonella enterica serovar Typhi is an intracellular anaerobic gram-negative bacterium, which causes typhoid fever in humans. The emergence of multi-drug resistance Salmonella typhi strains has become a significant issue leading to more difficulties in the management of disease especially in developing countries. High genetic variations among the field isolates may cause vaccination failure. Therefore, there is a need of conserved and immunogenic proteins as vaccine candidate for $S$. typhi. Recent studies have shown that outer membrane proteins (Omps) of Salmonella have been considered as immunogens for eliciting active/protective immune response against Salmonella and thus, have great potential to act as possible vaccine candidates for typhoid. In present study, Outer membrane proteins of Salmonella typhi were isolated by ultracentrifugation method followed by quantification by Lowry method and recovery was found to be $30 \mathrm{mg} / \mathrm{lit}$ approximately. Further Omps were analyzed by SDS-Polyacrylamide gel electrophoresis and nine proteins bands were observed ranging from small to medium size.

\section{Introduction}

Typhoid fever is a disease of human health concern caused by Salmonella enterica serovar Typhi, an important facultative gramnegative pathogen. It is a serious problem in most of the developing countries especially Southeast Asian countries, Africa and Latin America. Typhoid remains a public health problem with an estimated 22 million cases and 200,000 related deaths occurring globally every year (Crump et al., 2004). The emergence of multidrug resistance is making the situation more grimmer (Chau et al., 2007). Currently available vaccines for typhoid fever have few limitations like shortterm immunity, high cost, etc. and there is a need of better vaccine candidate. In last few 
years outer membrane proteins of Salmonella have been targeted for testing of their immunepotential (Hamid and Jain, 2010; Jha et al., 2015; Saxena et al., 2017) as these are considered to be conserved among the field isolates. Mainly two approaches have been used by various workers. Few of workers have targeted single Outermembrane protein (Jha et al., 2015; Prejit et al., 2013; Saxena et al., 2017) while others have used total Omps of Salmonella (Hamid and Jain., 2010; Meenakshi et al., 1999). In both the approaches, proteins have exhibited their immunepotential with various degree. Therefore, the present study was undertaken to isolate and characterize the Outer membrane proteins of Salmonella Typhi.

\section{Materials and Methods}

\section{Bacterial strain}

Pure culture of Salmonella enterica serovar Typhi (MTCC 733) was procured from CSIRInstitute of Microbial Technology, Chandigarh, India. The culture was revived in Luria Bertani broth and was tested by Salmonella specific PCR followed by biochemical characterization. Further, culture was maintained in Luria Bertani agar slants and glycerol stocks during the study.

\section{Outer membrane proteins preparation}

Outer membrane proteins of Salmonella typhi were isolated by method described by ChoiKim et al., (1991) with few modifications Single colony of $S$. typhi culture was inoculated in $2 \mathrm{ml}$ Luria Bertani broth and incubated at $37^{\circ} \mathrm{C}$ in incubator-shaker for 18 hrs. One ml of overnight culture was sub cultured in $500 \mathrm{ml}$ Luria Bertani broth and incubated at $37^{\circ} \mathrm{C}$ for $18 \mathrm{hrs}$. in incubatorshaker. Culture was centrifuged at 10,000 rpm for 10 minutes and pellet was obtained. Further, pellet was washed twice with
Phosphate buffer saline ( $\mathrm{pH}$ 7.2) and resuspended in 10mM HEPES buffer ( $\mathrm{pH}$ 7.4). Culture was sonicated by ultrasonication (20 cycles $(7.0 \mu)$ for 60 seconds followed by 30 seconds pause) and then centrifuged at $1700 \mathrm{xg}$ for 20 minutes. Supernatant was separated in a fresh tube and passed through $0.22 \mu$ filter. Filterate was ultra-centrifuged at $100,000 \times \mathrm{g}$ for 60 minutes at $4^{\circ} \mathrm{C}$. Pellet was collected and resuspended in $2 \mathrm{ml}$ of $2 \%$ Sodium lauryl sarkosinate in 10mM HEPES buffer followed by incubation at $37^{\circ} \mathrm{C}$ for one hour.

After incubation, cell lysate was again ultracentrifuged at $100,000 \mathrm{~g}$ for 60 minutes. Supernatant was discarded and pellet was resuspended in $500 \mu \mathrm{l}$ of Phosphate buffer saline (PBS). The protein was quantified by protein estimation method and protein profiling was determined by performing SDSPolyacrylamide gel electrophoresis using the method of Laemmli (1970).

\section{Quantification and profiling of Omps of S.typhi (MTCC 733)}

The protein was quantified by Lowry et al., 1951 protein estimation method using Bovine serum albumin as a standard and protein profiling was determined by performing SDSPolyacrylamide gel electrophoresis using the method of Laemmli (1970). The vertical slab gel electrophoresis apparatus (Atto, Japan) with glass plates of $14 \times 14 \mathrm{~cm}$ and spacer of $1.5 \mathrm{~mm}$ thickness was used for performing SDS-PAGE by discontinuous buffer system using $12 \%$ resolving gel and 5\% stacking gel.

\section{Results and Discussion}

The purity of culture was confirmed by biochemical characterization and Salmonellaspecific PCR. In biochemical characterization, culture was found to be MR+, VP-, and Urease- which is characteristic of Salmonella typhi. 
Outer membrane proteins of S. typhi MTCC 733 were isolated and quantified by Lowry method and recovery was found to be $30 \mathrm{mg} / \mathrm{l}$ approximately. Protein was further characterised by SDS-PAGE.

On SDS-Polyacrylamide gel electrophoresis analysis nine protein bands of approximately $20,26,30,32,36,39,42,47,49 \mathrm{KDa}$ in molecular weight size were observed. Among these bands five bands of molecular weight 42, 36, 30, 26 and 20KDa were major This showed that Omps were ranging from small to medium size, as the size of the proteins was ranging from $20-49 \mathrm{kDa}$. Our findings are very similar with findings of earlier workers as Muthiadin et al., (2015) reported the Omp profile of field isolate of Salmonella typhi in the same range of molecular weight as they reported the major bands of $25 \mathrm{KDa}, 35 \mathrm{KDa}$, 55 KDa. Similarly, Kumar et al., 2012 isolated and characterized the Outer membrane proteins of S. Gallinarum and reported the seven distinct protein bands among which 18, $38,40,43,45,50$ and $60 \mathrm{KDa}$ were found to be major protein bands. Though, earlier Hamid and Jain, 2008 reported the Outer membrane proteins of $S$. typhimurium ranging between 15-100 KDa, which is different from finding of other recent reports. The possible explanation is that Hamid and Jain, 2008 used different method (Foulaki et al., 1989) which is composed of lysis and dialysis of bacterial protein. Later on this method was replaced by ultracentrifugation method (Choi-Kim et al., 1991) (Fig. 1).

Fig.1 Isolation of Omps of Salmonella Typhi (M-Protein marker, 1-Outer membrane proteins of S.Typhi, 2- Whole cell proteins of Salmonella Typhi

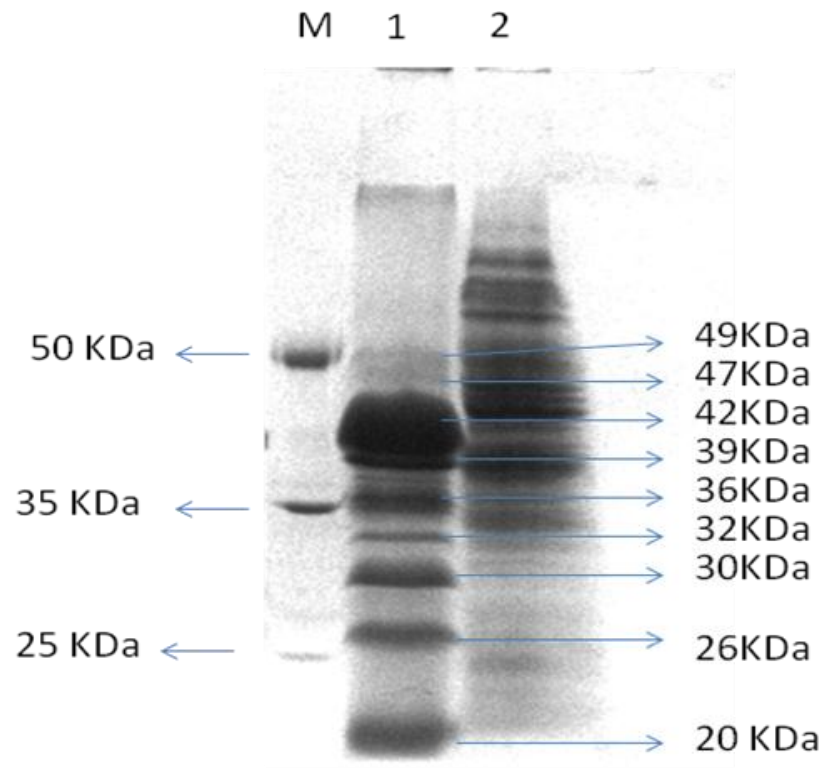

Salmonella is an important pathogenic organism, which causes disease in human and animals (Chui et al., 2004). In human beings it causes two types of disease. Typhoid fever caused by $S$. typhi and non-typhoidal salmonellosis mainly caused by $S$. typhimurium and S. enteritidis (Barquist et al.,
2013). Salmonella has high morbidity and mortality rate and multiple drug resistance which have been reported from most of the part of world has made the situation more difficult (Rahman et al., 2014; Kumar et al., 2013; Tamuly et al., 2012; Saxena et al., 2004). Therefore, the vaccination is the only 
viable option. Presently, available vaccines have few limitations such as they are costly and cannot be used for mass vaccination in developing countries like India (Sudharshan et al., 2014). Several approaches have been used for development of suitable vaccine such as mutant vaccine (Shippy et al., 2012), subunit vaccine (Hamid and Jain, 2010) and rDNA vaccine (Saxena et al., 2017). All these approaches have exhibited success with different level but still a potent protective immunogenic candidate could not be explored.

Outer membrane proteins of Salmonella are conserved proteins. Therefore, they can overrule the possibilities of vaccination failure due to high genetic variations (Yang et al., 2013). Outer membrane proteins of several serovars have been targeted for development of r-DNA vaccine against Salmonella and few of them have exhibited their immunepotential such as OmpC (Jha et al., 2015; Prejit et al., 2013). Omp 49 (Hamid and Jain., 2010) and Omp 28 (Saxena et al., 2017) but none of the single Omp could provoke complete protective immunity. Earlier in few studies, workers have used total Omps in place of single Omp and reported a better protection (Meenakshi et al., 1999; Hamid and Jain., 2010). Therefore, we isolated and characterized total Omps of Salmonella typhi. On basis of findings of earlier workers, we can conclude as Omps are conserved proteins and if they are used along with new generation adjuvant systems like Calcium phosphate nanoparticles (Tamuly and Saxena, 2012) they may be proven as an effective candidate for vaccine development against Salmonella Typhi.

\section{References}

Crump, J.A., Luby, S.P., Mintz, E.D. 2004. The global burden of typhoid fever. Bull. World Health Organ. 82:346-53.
Chau, T.T., Campbell, J.I., Galindo, C.M., Van Vinh Minh Hoang, N., et al. 2007. Antimicrobial drug resistance of Salmonella enterica serovar Typhi in Asia and molecular mechanism of reduced susceptibility to the fluoroquinolones. Antimicrobial agents and chemotherapy. 51(12): 4315-4323.

Hamid, N. and Jain, S.K. 2010. Immunogenic evaluation of a recombinant 49kilodaltonouter membrane protein of Salmonella Typhi as a candidate for a subunit vaccine against typhoid. J. Infect. Dis. Immun 2(2): 30-40.

Jha, R., Kumar, A., Saxena, A., Pandey, M., Kumar, R., Saxena, M.K. 2015. Heterogenous expression and functional evaluation of in silico characterized recombinant OmpC of Salmonella typhimurium as a functional poultry vaccine to eradicate zoonotic transmission. African Journal of Biotechnology 14(41): 2862-2870.

Saxena A, Kumar R, Saxena MK. 2017. Vaccination with Salmonella Typhi recombinant outer membrane protein 28 induces humoral but non-protective immune response in rabbit. Veterinary World 10(8): 946-949.

Prejit, Agarwal, R.K., Porteen, K., Dubal, Z.B., Karthikeyan, A., Singh, S., Biswas, R.2013. Evaluation of recombinant outer membrane protein based vaccine against Salmonella typhimurium in birds. Biologicals 41(3): 162-8.

Meenakshi, M., Bakshi, C.S., Butchaiah, G., Bansal, M.P., Siddiqui, M.Z., Singh, V.P. 1999. Adjuvanted Outer membrane protein vaccine protects poultry against infection with Salmonella enteritidis. Veterinary Research communication 23(2): 81-90.

Choi- Kim, K., Maheswaran, S.K., Felice, L.J and Molitor, T.W. 1991. Relationship between the iron regulated Omp and the Omp of in vivo grown Pasteurella multocida. Veterinary Microbiology 28(1):75-92. 
Laemmli, U.K. 1970. Cleavage of structural proteins during the assembly of the head of bacteriophage T4. Nature 227:680-685.

Lowry, O.H., Rosebrough, N.J., Farr, A.L. and Randall, R.J. 1951. Protein measurement with the Folin's phenol reagent. Journal of Biological Chemistry 193:265-75.

Muthiadin, C., Natsir, R., Agus, R., Nasrum, M., Dwiyanti, R., Sabir, M., Yasir, Y., Hatta, M. 2015. Identification and Characterization of Antigenic $36 \mathrm{KDa}$ Outer membrane protein (Omp) of Salmonella enterica serovar Typhi (S.typhi) from Makassar, South Sulawesi, Indonesia. American Journal of Biomedical Research 3(1): 9-12.

Kumar, A., Saxena, A., Tamuly, S., Kumar, R., Saxena, M.K. 2012. Immunological Characterisation of Outer membrane proteins (Omps) of Salmonella enterica serovar Gallinarum. J Immunol Immunopathol. 14(2): 105-109.

Hamid, N and Jain, S.K. 2008. Characterization of an outer membrane protein of Salmonella enterica serovar Typhimurium that confers protection against typhoid. Clin Vaccine Immunol 15: 1461-1471.

Foulaki, K., Gruber, W., and Schlecht, S. 1989. Isolation and immunological characterization of 55-kilodalton surface protein from Salmonella typhimurium. Infect Immun 57(5): 1399-1404.

Chiu, C.H., Su, L.H., Chu, C. 2004. Salmonella enterica serotype Choleraesuis: epidemiology, pathogenesis, clinical disease, and treatment. Clin Microbiol Rev 17(2):311-322.

Barquist, L., Langridge, G.C., Turner, D.J., Phan, M.D., Turner, A.K., Bateman, A., Parkhill, J., Wain, J., and Gardner, P.P.
2013. A comparison of dense transposon insertion libraries in the Salmonella serovars Typhi and Typhimurium. Nucleic Acids Research 41 (8): 45494564

Rahman, B.A., Wasfy, M.O., Maksoud, M.A., Hanna, N., Dueger, E. and House, B. 2014. Multidrug resistance and reduced susceptibility to ciprofloxacin among Salmonella enterica serovar Typhi isolated from the Middle East and Central Asia. Microbes New Infect 2 (4): 88-92.

Tamuly, S and Saxena M.K. 2012. Preparation of Calcium Phosphate Nanoparticles and Evaluation of their effects on muscle cells of rat. Current Science 102:610-612.

Saxena, M.K., Singh, V.P., Lakhchaura, B.D., Saxena, A., and Sharma, B. 2004. Detection of virulence in Indian isolates of Salmonella by polymerase chain reaction. Indian Journal of Biotechnology 3(1): 37-40.

Sudharshan, C.R. 2014. Clinical profile and antibiotic sensitivity pattern of Typhoid fever in patients admitted to pediatric ward in a rural teaching hospital. Int.J.Med Res and Health Sciences 3(2): 245-249.

Shippy, D.C., Fadl, A.A. 2012. Immunological characterization of a gidA mutant strain of Salmonella for potential use in a liveattenuated vaccine. BMC Microbiol 12:286.

Yang, Y., Wan, C., Xu, H., Aquilar, Z.P., Tan, Q., Xu, F., Lai, W., Xiong, Y. and Wei, H. 2013. Identification of an outer membrane protein of Salmonella enterica serovar Typhimurium as a potential vaccine candidate for salmonellosis in mice. Microbes Infect, 15(5): 388-398.

\section{How to cite this article:}

Yashpal Singh, Anjani Saxena, Rajesh Kumar and Saxena, M.K. 2018. Isolation and Protein Profiling of Outer Membrane Proteins (Omps) of Salmonella typhi. Int.J.Curr.Microbiol.App.Sci. 7(08): 2851-2855. doi: https://doi.org/10.20546/ijcmas.2018.708.300 